\title{
Déficit Del Autocuidado En El Adulto Mayor Con Diabetes
}

\section{Self-care deficit in the elderly with diabetes}

DOI: $10.46932 / \mathrm{sfjdv2n5-087}$

Received in: Oct 1st, 2021

Accepted in: Dec 30th, 2021

\section{Idely Yuraí Velasco-Molina}

Área Académica de Enfermería, Instituto de Ciencias de la Salud, Universidad Autónoma del Estado de Hidalgo, Pachuca 42160, Hidalgo, México

E-mail: ve340861@uaeh.edu.mx

\section{Ana Gabriela Hernández-Hernández}

Área Académica de Enfermería, Instituto de Ciencias de la Salud, Universidad Autónoma del Estado de Hidalgo , Pachuca 42160, Hidalgo, México

E-mail: he368461@uaeh.edu.mx

\section{Reyna Cristina Jiménez-Sánchez}

Área Académica de Enfermería, Instituto de Ciencias de la Salud, Universidad Autónoma del Estado de Hidalgo, Pachuca 42160, Hidalgo, México

E-mail: cristyji@hotmail.com

\section{Esther Ramírez Moreno}

Centro de Investigación Interdisciplinario, Área Académica de Nutrición, Instituto de Ciencias de la Salud, Universidad Autónoma del Estado de Hidalgo , Pachuca 42160, Hidalgo, México;

E-mail: esther_ramirez@uaeh.edu.mx

\section{Rosa María Baltazar-Tellez}

Área Académica de Enfermería, Instituto de Ciencias de la Salud, Universidad Autónoma del Estado de Hidalgo, Pachuca 42160, Hidalgo, México

E-mail: rosa_baltazar@uaeh.edu.mx

\section{Olga Rocío Flores-Chávez}

Área Académica de Enfermería, Instituto de Ciencias de la Salud, Universidad Autónoma del Estado de Hidalgo , Pachuca 42160, Hidalgo, México

E-mail: ofloresc@uaeh.edu.mx

\section{José Javier Cuevas-Cansino}

Área Académica de Enfermería, Instituto de Ciencias de la Salud, Universidad Autónoma del Estado de Hidalgo, Pachuca 42160, Hidalgo, México

E-mail: jose_cuevas@uaeh.edu.mx

\section{José Arias-Rico}

Área Académica de Enfermería, Instituto de Ciencias de la Salud, Universidad Autónoma del Estado de Hidalgo , Pachuca 42160, Hidalgo, México

E-mail: jose_arias@uaeh.edu.mx 


\begin{abstract}
Older adults who go through a situation of family abandonment tend to suffer from sadness, loneliness or even worse depression, which leads to various situations in their daily lives. Most older adults suffer from type 2 diabetes mellitus, living with this pathology requires multiple care and interventions by the person himself and with the support of the rest of the family, in order to provide adequate routine care for the patient; That is why when there is family neglect, patients lose interest in taking care of themselves and tend to fall into a routine. In the present study, the self-care deficit in older adults was identified. It is a study of non-experimental quantitative design, cross-sectional, correlational, since it is carried out at a specific time and the variables do not have a control. The population is older adults with diabetes pathology, inhabitants of a day house in the state of Hidalgo, Mexico. The sample consisted of 30 people by availability. A survey was conducted among the elderly, only patients living with diabetes pathology to make an analysis of the care performed. As results, it was obtained that approximately $3 \%$ of older adults in a home for the elderly in Pachuca de Soto, Hidalgo, carry out proper self-care in their daily activities. A third of older adults have a self-care deficit, it is associated with different situations, starting with non-adherence to treatment and unhealthy habits. The majority of older adults have self-care deficits and therefore tend to be complicated by the pathologies they suffer, it is likely that their blood glucose levels are elevated, as well as the appearance of xerosis on the skin on the feet and hands; To correct this behavior, it is suggested to carry out workshops to teach the correct way to carry out adequate care, as well as to encourage adherence to treatments, carry out a good diet and exercise regularly in order to improve their health and extend life expectancy.
\end{abstract}

Keyword: Diabetes, déficit, self-care, older adults.

\title{
RESUMEN
}

Los adultos mayores que atraviesan por una situación de abandono familiar, tienden a padecer tristeza, soledad o aún peor depresión, que conlleva a diversas situaciones en su vida cotidiana. La mayoría de los adultos mayores padecen diabetes mellitus tipo 2, vivir con esta patología requiere de múltiples cuidados e intervenciones por parte de la persona misma y con apoyo del resto de la familia, para poder llevar un cuidado habitual adecuado al paciente; es por ello que cuando existe un abandono familiar los pacientes pierden el interés de cuidarse y tienden a caer en la rutina. En el presente estudio se identificó el déficit del autocuidado en los adultos mayores. Es un estudio de diseño cuantitativo no experimental., transversal, correlacional, ya que se efectúa en un momento específico y las variables no tienen un control. La población, son adultos mayores con patología de diabetes, habitantes de una casa de día del estado de Hidalgo, México. La muestra fue de 30 personas por disponibilidad. Se realizó una encuesta entre los adultos mayores, solo a los pacientes que viven con la patología de diabetes para hacer un análisis de los cuidados realizados. Como resultados se obtuvo que un 3\% aproximadamente de adultos mayores de una casa de la tercera edad en Pachuca de Soto, Hidalgo, realizan un correcto autocuidado en sus actividades diarias. Más de la tercera parte de adultos mayores tienen déficit de autocuidado, se asocia a diferentes situaciones iniciando por el no apego al tratamiento y a hábitos poco saludables. La mayoría de los adultos mayores tienen déficit del autocuidado y por lo tanto suelen complicarse en las patologías que padecen, es probable que sus niveles de glucosa en sangre se vean elevados, al igual que la aparición de xerosis en la piel en pies y manos; para corregir esta conducta se sugiere realizar talleres para enseñar la forma correcta de realizar los cuidados adecuados, así como fomentar el apego al tratamientos, llevar a cabo una buena alimentación y a ejercitarse de manera regular para así mejorar su salud y alargar la esperanza de vida.

Palabras Claves: Diabetes, déficit, autocuidado, adultos mayores. 


\section{INTRODUCCIÓN}

El término diabetes mellitus (DM) define alteraciones metabólicas de múltiples etiologías caracterizadas por hiperglucemia crónica y trastornos en el metabolismo de los hidratos de carbono, las grasas y las proteínas, resultado de defectos en la secreción de insulina, en la acción de la misma o en ambas (Ramos-Manchego y cols. 2020). Según las estimaciones, 422 millones de adultos en todo el mundo tenían diabetes en 2014, frente a los 108 millones de 1980. Se prevé que para el año 2040 esta cifra habrá aumentado hasta alcanzar los 642 millones de afectados (Pereyra Gonzales 2020).

La prevalencia mundial (normalizada por edades) de la diabetes casi se ha duplicado desde ese año, pues ha pasado del 4,7\% al 8,5\% en la población adulta. Ello supone también un incremento en los factores de riesgo conexos, como el sobrepeso o la obesidad. En la última década, la prevalencia de la diabetes ha aumentado más deprisa en los países de ingresos bajos y medianos que en los de ingresos altos. (Collantes Delgado 2019)

En 2012, la diabetes provocó 1,5 millones de muertes. Un nivel de glucosa en la sangre superior al deseable provocó otras 2,2 millones de muertes, al incrementar los riesgos de enfermedades cardiovasculares y de otro tipo. Un 43\% de estos 3,7 millones de muertes ocurren en personas con menos de 70 años. El porcentaje de muertes atribuibles a una glucemia elevada o a la diabetes en personas menores de 70 años de edad es superior en los países de ingresos bajos y medios que en los de ingresos altos. (Informe Mundial sobre la Diabetes, OMS, 2016)

La diabetes tipo 2 es la que se observa más comúnmente en adultos mayores, pero se observa cada vez más en niños, adolescentes y adultos jóvenes por el incremento en los niveles de obesidad, sedentarismo y una dieta inadecuada. (IDF Diabetes Atlas 8th Edition 2017); tanto en la Diabetes tipo 1 como en la tipo 2, diversos factores ambientales y genéticos pueden resultar en la pérdida progresiva de la función y/o la masa de células beta que se manifiesta clínicamente como hiperglucemia. Una vez que la hiperglucemia aparece, los pacientes con cualquier forma de diabetes se encuentran en riesgo de desarrollar las mismas complicaciones crónicas, aunque las tasas de progresión pueden diferir. (Standards of Medical Care in Diabetes 2018).

La diabetes tipo 2, previamente conocida como Diabetes no insulinodependiente o diabetes del adulto, representa el 90-95\% de todos los casos de Diabetes. Esta forma engloba a los individuos que tienen una deficiencia de insulina relativa y que presentan resistencia periférica a la insulina. Estos individuos, al menos de inicio, y muy comúnmente durante el resto de su vida, no necesitan tratamiento con insulina para sobrevivir. Existen diversas causas de Diabetes tipo 2. Aunque no se conocen con exactitud las etiologías específicas, no ocurre una destrucción autoinmune de células beta, y los pacientes no tienen alguna de las otras causas conocidas de diabetes. La mayoría de estos pacientes presentan sobrepeso u obesidad. El exceso de peso causa por sí mismo un grado de resistencia a la insulina. Los 
pacientes con Diabetes que no tienen sobrepeso u obesidad pueden tener un incremento en el porcentaje de grasa corporal distribuida predominantemente en la región abdominal. (Standards of Medical Care in Diabetes 2018).

Se han descrito múltiples factores de riesgo. Dentro de los más importantes están: La presencia de Diabetes Mellitus tipo 2 en familiares de primer grado, tener hipertensión arterial, sedentarismo, un índice de masa corporal mayor de 25, y una circunferencia de cintura de más de $102 \mathrm{~cm}$ en hombres y 88 en mujeres. (Pérez A. 2014) (Standards of Medical Care, ADA 2018)

Para el año 2014 la Federación Mexicana de Diabetes determinó que existen 4 millones de personas con este padecimiento en nuestro país. Los estados de mayor prevalencia son: la Ciudad de México, Nuevo León, Veracruz, Tamaulipas, Durango y San Luis Potosí. Esto representó un gasto importante de 3,430 millones de dólares (68,600,000 millones de pesos) al año en su atención y en el manejo de las complicaciones. (Federación mexicana de Diabetes, A.C. 2014) Se estima que, en nuestro país, 1 de cada 11 adultos vive con diabetes, de esta cifra, las personas con Diabetes Mellitus tipo 2, tienen una edad entre 40 y 59 años. De éstos, 5\% no tienen un diagnóstico y el 77\% vive en países con ingresos medios y bajos, originando, a su vez, 548 millones de dólares del gasto sanitario en el 2012. (Federación Mexicana de Diabetes, 2014)

Los adultos mayores que atraviesan por una situación de abandono familiar, tienden a padecer tristeza, soledad o aún peor depresión, que conlleva a diversas situaciones en su vida cotidiana. La mayoría de los adultos mayores padecen diabetes mellitus tipo 2, vivir con esta patología requiere de múltiples cuidados e intervenciones por parte de la persona misma y con apoyo del resto de la familia, para poder llevar un cuidado habitual adecuado al paciente; es por ello que cuando existe un abandono familiar los pacientes pierden el interés de cuidarse y tienden a caer en la rutina

"Autocuidado: Conjunto de acciones intencionadas que realiza o realizaría la persona para controlar los factores internos o externos, que pueden comprometer su vida y desarrollo posterior" (Orem, 1969).

El autocuidado se refiere a aquellas actividades que realizan los individuos, familia o comunidad, con el propósito de promover la salud, prevenir la enfermedad, limitarla cuando existe o restablecer la salud. Como todo proceso social, la educación para el autocuidado debe permitir al individuo definir su situación de salud, identificar los problemas a medida que éstos surgen, encontrar soluciones, ya sea cuidando de sí mismo, teniendo atención adecuada o realizando acciones sociales dirigidas hacia el problema específico. La educación debe comenzar donde se encuentran las personas, esto supone realizarlas en base a las conductas de autocuidado ya presentes en la comunidad. Sería difícil y probablemente poco satisfactorio, implementar un cambio por necesario que éste sea sin considerar la forma en que la persona satisface sus necesidades diarias de autocuidado. No considerar el ambiente de 
las personas, sus estilos de vida, sus formas de relacionarse, sus creencias y valores, impide lograr cambios de conductas y adherencia a las acciones de autocuidado. Valorar estas condiciones hace que los profesionales de la salud asignen gran importancia a ciencias como psicología, sociología y antropología que ayudan a conocer y comprender las diferentes formas del comportamiento humano.

El objetivo de este trabajo fue identificar los hábitos de autocuidado de los adultos mayores que padecen diabetes en una casa de la tercera edad del estado de Hidalgo.

\section{MÉTODO}

\subsection{DISEÑO Y TIPO DE INVESTIGACIÓN}

Es un estudio de diseño cuantitativo no experimental., transversal, correlacional, ya que se efectúa en un momento específico y las variables no tienen un control.

\subsection{POBLACIÓN Y MUESTRA}

La población muestra, son adultos mayores con patología de diabetes habitantes de la casa de la tercera edad del estado de Hidalgo, México. La muestra fue de 30 personas por disponibilidad.

\subsection{INSTRUMENTO}

Se realizó una encuesta que consta de 20 preguntas, las primeras 2 preguntas son relacionadas a la edad y el sexo de los participantes, las siguientes 5 preguntas van encaminadas a determinar el tipo de alimentación que llevan a cabo durante 7 días, las 2 preguntas siguientes son relacionadas al ejercicio que realizan los adultos mayores en los 7 días evaluados, las siguientes 2 preguntas se relacionan a las veces que se hizo medición de azúcar en sangre durante la semana a evaluar, 4 preguntas son referidas al cuidado de los pies realizado habitualmente y las 3 próximas preguntas van dirigidas a la ingesta de medicamentos adecuada, para finalizar 2 preguntar relacionadas al hábito de fumar. Se aplicó el instrumento durante 2 días con los adultos mayores se explicó la forma en que se desde responder. El cuestionario fue elaborado por el equipo de investigación en el proceso.

\subsection{PROCEDIMIENTO}

La recolección de datos se llevó a cabo mediante la aplicación del instrumento para determinar el nivel de autocuidado que aplica cada paciente en su vida cotidiana.

\section{ANÁLISIS ESTADÍSTICO}

Se utilizó el paquete estadístico SPSS V.21 para Windows. Mediante el análisis del comportamiento del estudio tras la aplicación de nuestro instrumento "Actividades de Autocuidado en 
Diabetes (SDSCA)", el cual busca valorar el grado del déficit de autocuidado en el adulto mayor con Diabetes, relacionando las dos variables para poder obtener un resultado adecuado. El protocolo fue revisado por el comité de investigación del área académica de enfermería.

\section{RESULTADOS}

El universo de este estudio estuvo conformado por 30 adultos mayores, de los cuales el $50 \%$ fueron del sexo femenino y el $50 \%$ masculino.

En la Figura 1 se observa las edades de los pacientes a los que se les aplicó el cuestionario.

Figura 1 Variables sociodemográficas

\begin{tabular}{|c|c|}
\hline Edad & Porcentaje \\
\hline 60 a 70 & $6.7 \%$ \\
70 a 80 & 56.7 \\
80 a 90 & 36.7 \\
\hline
\end{tabular}

Fuente: Cuestionario "autocuidado en adultos mayores".

En la Figura 2 se observa los resultados del resto del instrumento en los que se muestra el nivel de déficit del autocuidado que se tiene entre los encuestados, alrededor del 13\% de los adultos mayores entrevistados son los que llevan un cuidado adecuado en todos los ámbitos y tienen un apego a el tratamiento indicado. Por el contrario el $87 \%$ de los encuestados presentan déficit en los cuidados realizados, así como, el desapego a tratamiento farmacológico y procedimientos médicos.

Figura 2: hábitos diarios, cuidados realizados y apego al tratamiento farmacológico

\begin{tabular}{|c|c|c|c|c|c|c|c|c|c|}
\hline & Rubroldías & 1 & 2 & 3 & 4 & 5 & 6 & 7 & Total \\
\hline \multirow{3}{*}{ Alimentación } & Régimen de alimentación Saludable & 0 & 0 & 7 & 6 & 12 & 21 & 54 & 100 \\
\cline { 2 - 8 } & Consumo de Frutas y Verduras & 0 & 0 & 0 & 15 & 15 & 20 & 50 & 100 \\
\cline { 2 - 8 } & Consumo de Carnes rojas o Lácteos & 44 & 30 & 10 & 7 & 6 & 2 & 1 & 100 \\
\cline { 2 - 8 } & Consumo de Carbohidratos & 50 & 34 & 7 & 6 & 2 & 1 & 0 & 100 \\
\hline \multirow{3}{*}{ Actividad física } & Actividad diaria mínima de 30 min. & 10 & 12 & 68 & 10 & 9 & 0 & 1 & 100 \\
\cline { 2 - 8 } & Chequeo de Glucosa & 3 & 10 & 10 & 24 & 16 & 27 & 10 & 100 \\
\hline \multirow{3}{*}{ Cuidado de Pies } & Chequeo de Pies & 0 & 0 & 0 & 0 & 1 & 10 & 89 & 100 \\
\cline { 2 - 8 } & Inspección de la parte interna de los zapatos & 26 & 39 & 15 & 10 & 3 & 2 & 5 & 100 \\
\cline { 2 - 8 } & Lavado de pies & 0 & 0 & 0 & 0 & 1 & 10 & 89 & 100 \\
\cline { 2 - 8 } & Secado entre los dedos después del lavado de pies & 0 & 0 & 0 & 0 & 8 & 12 & 80 & 100 \\
\hline \multirow{3}{*}{ Medicamentos } & Toma de medicamentos & 0 & 0 & 4 & 12 & 9 & 30 & 45 & 100 \\
\cline { 2 - 8 } & Ministración de insulina & 2 & 4 & 4 & 13 & 9 & 28 & 40 & 100 \\
\cline { 2 - 7 } & Apego a tratamiento farmacológico & 0 & 0 & 4 & 12 & 9 & 30 & 45 & 100
\end{tabular}

Fuente: Cuestionario "déficit del autocuidado en adultos mayores". 


\section{DISCUSIÓN}

La presente investigación logró identificar las distintas deficiencias en el cuidado del adulto mayor con diabetes, identificando distintos aspectos y con qué frecuencia son realizadas durante una semana.

Existe un bajo nivel de conocimientos sobre autocuidado, se cree que se tiene un mayor conocimiento sobre el tratamiento el cual está ligado al tiempo de evolución de la enfermedad, llegaron a la conclusión que a mayor tiempo de vivir con la enfermedad mayor conocimiento para tratar la misma de una forma adecuada, en cuestión de prevención y promoción el país está muy atrasado en comparación con otros países. (Pousa Reis M. A. 2017)

\section{CONCLUSIONES}

Por lo menos la tercera parte de la población encuestada reveló, que los pacientes que viven con diabetes dejan de realizar las acciones de autocuidado en el tratamiento y control de la diabetes, se piensa que al no tener un familiar o persona responsable que brinde tales cuidados, los pacientes dejan de realizarlos por olvido o por aburrimiento a la monotonía de la enfermedad. Se estima que el $13 \%$ de la población lleva adecuadamente los cuidados necesarios para la enfermedad y tienen una probabilidad más alta de alargar el tiempo de vida. 


\section{REFERENCIAS}

[1] Jiménez CA, Aguilar SCA, Rojas MR, Hernández AM. Diabetes mellitus tipo 2 y frecuencia de acciones para su prevención y control. Scielo.2013;55:137-143.

[2] Romero BI, Santos MA, Aparecida MT, Zenetti ML. Autocuidado de personas con Diabetes Mellitus atendidas en un servicio de urgencia en México. Rev. Latino-Am Enfermaren. 2010;18(6):123129

[3] Abellán G, Abizanda P, Alastuey C, Tratado de Geriatría para residentes. Sociedad Española de Geriatría y Gerontología (SEGG) [Internet]. [Consultado el 20 de Julio 2018] Madrid: International Marketing \& Communication, S.A. (IM\&C), 2014. Recuperado de https://bit.ly/3rXKdn8

[4] Grupo de trabajo de la Guía de Práctica Clínica sobre Diabetes tipo 2. Guía de Práctica Clínica sobre Diabetes tipo 2. Madrid: Plan Nacional para el SNS del MSC. Agencia de Evaluación de Tecnologías Sanitarias del País Vasco; 2008. Guías de Práctica Clínica en el SNS: OSTEBA No 2006/08

[5] de Jesús Ramos-Mancheno, A. D. (2020). Trastornos metabólicos no congénitos como causa de diabetes mellitus tipo 2. Dominio de las Ciencias, 6(5), 111-127.

[6] Diagnóstico y Tratamiento Farmacológico de la Diabetes Mellitus Tipo 2 en el Primer Nivel de Atención. Guía de Evidencias y Recomendaciones: Guía de Práctica Clínica. México, Instituto Mexicano del Seguro Social; 2018. Disponible en: https://bit.ly/3lsvI8y

[7] Pereyra González, I. (2020). Medidas antropométricas al nacer, en el primer año y presencia de alteraciones metabólicas que predicen diabetes mellitus II (Doctoral dissertation).

[8] Collantes Delgado D.G. Gonzales Manoslava L. (2019) Hipertensión arterial y diabetes mellitus en adultos de la ciudad de chota 2018. Disponible en: https://bit.ly/3tzqgn5

[9] Pousa Reis M. A. (2017) Nivel de conocimiento de autocuidado en el paciente diabético tipo 2 de la UMF No. 8 de Aguascalientes. Disponible en: https://bit.ly/3bYv7IA 